SHORT REPORT

\title{
A regional approach to improving night time supervision of accident and emergency departments
}

\author{
P Jaye, M W Beckett, A Parfitt
}

Emerg Med J 2004;21:65-66

$\mathrm{T}$ he objective of this study was to assess the feasibility of senior cover provided to a training region, covering multiple sites, between the hours of 0000 to 0800 by a distant specialist registrar using the facsimile and telephone. Senior cover is already available to all NHS hospitals within the UK. Unfortunately this is often provided by consultants on unreasonable rotas. Twenty four hour middle grade cover happens rarely. The case mix remains complex and varied however out of normal working hours. North West Thames represents a comparatively compact training region in emergency medicine however staffing levels mean that only two of eight hospitals are able to provide access to a middle grade opinion 24 hours a day. Dale et $a l^{1}$ have shown that what SHOs desire is access to middle grade advice. Senior night time cover is becoming a prerequisite for safe effective supervision of junior doctors practising emergency medicine. Staffing, budget, and recruitment conspire to preclude this dream from reality.

Our hypothesis was that a solitary SpR possibly rotating from the pool within region is able to effectively safely and from a cost viewpoint improve existing services. He was based at one hospital and was available for face to face removed from patient consultation while the other hospitals in region could telephone and fax ECGs.

\section{METHOD AND RESULTS}

This prospective study was undertaken over a two month period between October and November 2000. Over three periods of five consecutive nights, night time cover was provided to six hospitals within the North Thames Area, all of these hospitals being served by the regional specialist registrar training programme. Only on one of these sites were specialist registrars available resident on site on call. Each of these hospitals had middle grade cover (specialist registrar or staff grade) until 2200 or 0000 hours. Normal cover was maintained at each hospital during this period.

At the hospital where the SpR was resident he was awake and provided "face to face" consultation advice and opinion on radiograph and electrocardiogram interpretation but did not enter into contact with patients. Facsimile allowed for ECG interpretation at the remaining hospitals. The study was publicised in advance at the regional SpR training days and locally at each hospital by means of a session at the SHO teaching programmes and feedback sessions were conducted during the study. The nights the SpR worked the hospitals were contacted and the senior house officers and nursing staff on duty were informed of his availability and the service on offer reiterated. Telephone and fax numbers were redistributed.

Before each consultation with the SpR, SHOs completed a questionnaire (see journal web site http://www.emjonline. com/supplemental). This included patient details and the management the SHO considered appropriate before the consultation had senior advice not been available. They had been instructed at the teaching sessions and on the night that this should not be communicated to the SpR. The SpR recorded the type of consultation and the outcome on a personal computer. The differences between the SHO management plan and that of the SpR were compared and assessed for their clinical and administrative outcome and significance. Each patient episode was followed up to assess their eventual outcome. Patients were followed up by inspection of GP letter and checking for re-attendance at any of the hospitals participating in the study.

Differences between the two outcomes were then scored for their clinical and administrative significance by three separate consultants blinded to the opinion of their peers.

Administrative changes included:

- Avoiding disturbing the on call team for an opinion. That is, the consultation with the SpR negated the need to wake the on call team and ask their advice, for example, an elderly fit gentleman with haematuria- "What investigation does he require?"

- Avoiding disturbing A\&E senior for opinion at home. For example, concern regarding the management of an alleged rape.

- Avoiding unnecessary outpatient appointment—bringing a patient back too early for assessment with a soft tissue injury to the knee.

- Hastening the decision making process but no real change - a child with haematuria for whom the SHO was waiting for results before referring.

Clinical changes included:

- Significant changes in treatment administered by SHOfor example, SHO wanting to thrombolyse a patient inappropriately from an ECG reviewed by facsimile.

- An urgent referral advised and the patient admitted2 year old re-attender with experienced mum admitted overnight.

In total 168 consultations were recorded over the period of 15 nights. Forty four per cent were face to face and 56\% were telephone consultations. A change in clinical care was made in $47 \%$ of the face to face consultations and $43 \%$ of the telephone consultations. A change to administrative care was made in $56 \%$ of the face to face consultations and $60 \%$ of the telephone consultations.

The average duration of consultation was 7 min $32 \mathrm{~s}$ for the face to face consultations and $6 \mathrm{~min} 48 \mathrm{~s}$ for the telephone consultations (table 1).

\section{DISCUSSION}

Senior A\&E cover is already available at night to all NHS hospitals within the UK. Unfortunately this cover is provided in many hospitals via consultants often on one in two or three rotas. Understandably there is a high threshold for its use and this service could be supplementary. The region 


\section{Table 1 Consultations by specialty}

\begin{tabular}{ll}
\hline Specialty & Number of consultations \\
\hline Medicine & 80 \\
Paediatrics & 10 \\
Orthopaedics & 23 \\
Plastics & 6 \\
ENT & 6 \\
Surgery/urology & 19 \\
Ophthalmic & 4 \\
Gynaecological & 7 \\
A\&E & 13 \\
\hline
\end{tabular}

would be able to rotate SpRs through a week of such nights for three periods a year. Using eight minute consultations the registrar could take a maximum of 60 a night. Economic savings would include avoidance of outpatient appointments and inappropriate investigation and admission and in extreme cases possible medicolegal costs.

In conclusion, night time cover to A\&E SHOs between the hours of 0000 to 0800 in North West Thames can be safely provided by an SpR remotely covering multiple sites via a telephone and fax. This cover produces not only administrative but also clinical improvements in patient care. Provided in this manner it represents an affordable compromise with a demonstrable change to the quality of care.
Within a training region as compact as ours it affords a continuity and quality of care that is otherwise or not yet possible or financially viable.

\section{CONTRIBUTORS}

M Beckett, P Jaye, and A Parfitt conceived the idea. P Jaye conducted the project. P Jaye and A Parfitt wrote the report.

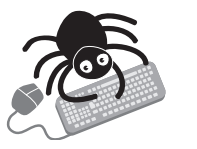

A copy of the questionnaire used in this study is available on the journal web site (http://www. emjonline.com/supplemental).

\section{Authors' affiliations}

P Jaye, M W Beckett, A Parfitt, Department of Accident and Emergency, West Middlesex Hospital, Isleworth, UK

Correspondence to: Dr A Parfitt, St Thomas' Hospital, Lambeth Palace Road, London SEI 7EH, UK; a@parfitt.org

Accepted for publication 3 June 2003

\section{REFERENCE}

1 Dale J, Williams S, Wellsesley A, et al. Training and supervision needs and experience: a longitudinal cross sectional survey of accident and emergency department senior house officers. Postgrad Med J 1999;75:86-9. 